\title{
The Free-Swimming Device Leakage Detection in Plastic Water-filled Pipes through Tuning the Wavelet Transform to the Underwater Acoustic Signals
}

\author{
Dileep Kumar ${ }^{1,3, *}$, Dezhan Tu ${ }^{1}$, Naifu Zhu ${ }^{1}$, Reehan Ali Shah ${ }^{2,4}$, Dibo Hou ${ }^{1}$ \\ and Hongjian Zhang ${ }^{1}$ \\ 1 State Key Laboratory of Industrial Control Technology, College of Control Science and Engineering, \\ Zhejiang University, Hangzhou 310027, China; 21632031@zju.edu.cn (D.T.); znf@zju.edu.cn (N.Z.); \\ houdb@zju.edu.cn (D.H.); hj_zhang@zju.edu.cn (H.Z.) \\ 2 Institute of Artificial Intelligence, College of Computer Science, Zhejiang University, \\ Hangzhou 310027, China; rehanalishah@zju.edu.cn \\ 3 Department of Electronic Engineering, University College of Engineering and Technology, Islamia \\ University of Bahawalpur, Punjab 63100, Pakistan \\ 4 Department of Computer Systems Engineering, University College of Engineering and Technology, Islamia \\ University of Bahawalpur, Punjab 63100, Pakistan \\ * Correspondence: 11332042@zju.edu.cn; Tel.: +86-1325-102-3837
}

Received: 25 July 2017; Accepted: 19 September 2017; Published: 23 September 2017

\begin{abstract}
The conventional fixed acoustic sensors leak detection methods have been demonstrated to be very practical for locating leakages in water distribution pipelines. However, these methods demand proper installation of sensors, and therefore cannot be implemented on buried long water distribution pipelines for condition assessment, early leak detection, and the estimation of leak size effect. Due to these limitations, a free-swimming device is developed. The free-swimming device with the potential of high acoustic sensitivity is capable of detecting the small underwater leakages in the plastic water-filled pipes. Despite the fact that a number of factors influence the underwater acoustic signals, such as water flow noise. Therefore, the interpretation of the leakage and influence of leakage size is considerably challenging from the underwater measured signals. The new method is proposed for reliable leakage detection by tuning the wavelet transform to underwater water acoustic signals. In this method, firstly, Short-Time Fourier Transforms (STFT) of underwater acoustic signals over a relatively long time-interval is monitored to capture the leakage-signals signature. The captured signals efficiently lead in the selection of mother wavelet (tuned wavelet) for the excellent signal localization in the time-frequency domain. Finally, the acoustic signals are analyzed in the tuned wavelet transform to detect the events. In this paper, the practical application of the proposed method, the controlled experiments are designed, and acoustic signals are collected from an experimental setup by launching the free-swimming device. The measured acoustic signals are used to identify the leakage-signals signature from unwanted interfering signals (instantaneous pipe vibrations, water flow noise, pipe's natural frequencies, and background noise). The evaluation of results validated that the free-swimming device and the tuned wavelet transform together can efficiently lead to reliable underwater leakage detection, as well as the influence of the leakage size in plastic water-filled pipes.
\end{abstract}

Keywords: acoustic sensors; acoustic signals; free-swimming device; localization; leakage detection; tuned wavelet

\section{Introduction}

There is a number of leading causes of leakages in the water distribution pipeline system, such as hazardous materials, the lifespan of pipe materials, severe environmental changes, and so forth. Leaks 
cannot be taken only into the account of water loss, or socioeconomic losses; it also allows harmful contaminants into the water which causes potential risk to people health. It is reported that globally four billion people in 97 countries are facing severe water scarcity problem at least one month/year and nearly half of the population (two billion) belong to China, India, and Pakistan [1]. This analysis specifies the increasing gap between demand and supply of potable water that can hamper the urban development and also includes the financial losses and possible risk to peoples' health. The research concludes that a water pipeline system still demands extremely efficient water management [2] to differentiate and fix faults and leaks to protect the environment and bridge the increasing breaches between demand and supply of potable water in urban areas.

In the past 20 years, a number of researchers have proven that the acoustic methods can well identify leakages from liquid or gas-filled pipeline systems. It has also confirmed that acoustic emission techniques are often enormously faster than traditional non-acoustic methods, such as ultrasonic, infrared thermography, tracer gas, electromagnetic scanning, and ground penetrating radars [3-5]. Conventional fixed acoustic methods are focused on longitudinal deployment, and due to the availability of various types of water pipe materials with extensive properties, these methods also demand a proper placement of sensors regarding the type of material. Sensor-to-sensor spacing on metal pipes can be between 200 to $500 \mathrm{~m}$, and in plastic, approximately up to $100 \mathrm{~m}$ due to low vibrating frequencies. As sensors placement distance becomes shorter and shorter, the more reliable leak detection and localization will be $[6,7]$. Experimentally, it is also authenticated that correlation-based methods are more reliable for the identification of leakage rather than the other methods [8,9]. Since these permanent leak detection techniques are based on continuous monitoring acoustic sensors, operational change is not possible [10]. It is very challenging to find small leaks regardless of their location in the pipeline, and the deployment cost is also high. Ground surface listening devices almost directly locate leak position. However, these methods are also not practically applicable for long and underground transmission pipelines [11].

Though the aforementioned permanent acoustic sensor techniques are based on continuous monitoring, they may not be very cost efficient, unable to find small leaks, and cannot apply for early leak detection in long distance and underground water pipeline. Unidentified small leaks can damage the environment, possibly turning into severe failures and resulting in very high maintenance costs. Early leak detection in hazardous pipe materials can reduce the product loss and related losses. Hence, due to a variety of pipe materials, different types of leakages and geographical positions of deployed pipeline systems, it significantly demonstrates that a single method either hardware-based or software-based cannot be applicable in all circumstances [4]. Therefore, to overcome these issues, a free-swimming acoustic device is developed [11-13], as shown in Figure 1. An acoustic, free-swimming leakage detection device is not a substitute for all of the existing technologies, but a compliment to the present water leak detection systems. It allows for the operators to choose the technology according to the need and site demand. The device consists of acoustic sensors that are rolled through the flow of the water and that measure the acoustic events in diameter of any pipe material.

Underwater detection of the acoustic signals generated by leaks was one of the primary challenges in designing the device. Therefore, a large number of acoustic sensors were tested to achieve a high acoustic sensitivity. As a result of a high acoustic sensitivity, the device is capable of detecting leaks with water flow noise and $100 \%$ coverage of inline inspection. The shape of the device is spherical, and smaller in size than the diameter of the pipeline that supports it to traverse inside the water-filled pipes easily. The free-swimming propelling device in water lines might be problematical so as to make the operation easier; pig traps can be utilized to inject and receive the device. 


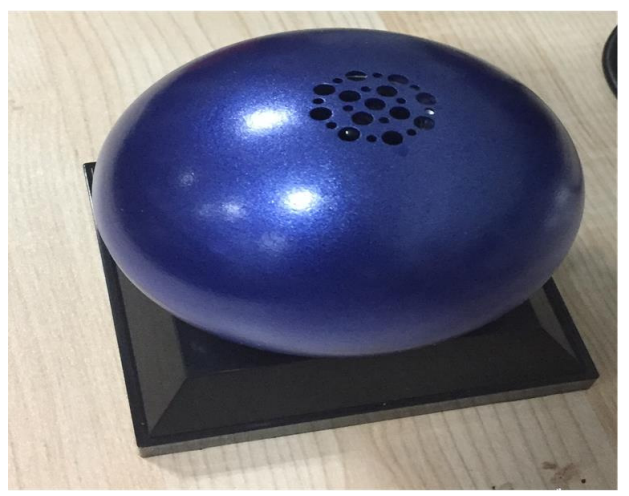

Figure 1. Free-swimming device.

There also exists other in-line pipe studies and technologies, such as mobile/wireless sensor networks design that is based on a group of mobile sensors nodes that work mutually according to a pre-scheduled order $[14,15]$, and nodes traverse with the water flow regardless of the pipeline material. The measured data can be collected by interacting with sensors through wireless access points outside of the pipes or by capturing the sensors physically for further numerical analysis $[16,17]$. Due to the limitations of sensors networks such as poor RF transmission, lack of maintainability, replacing, or repairing the nodes is extremely costly and hard to implement on existing pipeline structure, it cannot be practically deployed in long and underground branched water distribution systems [18,19].

Free-swimming correlated acoustic technologies, i.e., the Smartball and Sahara [20,21], are also capable of detecting small leakages in water pipelines. However, in both cases, it is hard to interpret underwater measured acoustic signal events due to variations of the surroundings parameters, signal similarities, and the stochastic nature of events $[9,22]$. Particularly in the case of small leaks, signal features completely hide under the impact of factors as mentioned above, and the leak size effect could not become apparent. A number of similar, free-swimming technologies and studies exists [22-25], but no study has focused on the investigation of underwater events. The free-swimming acoustic device with events analysis capability can be a very reliable and robust underwater acoustic leak detection technology which is a key focus of this research.

Since processing of the acoustic signal in the time and frequency domain can generate useful information, simultaneous time-frequency is demonstrated to be very efficient. The Short-Time Fourier Transform (STFT) is employed in the context of time-frequency analysis; although simple to use, the accuracy of STFT is confined to the window size. Hence, STFT is not particularly popular for the acoustic signal investigation in water distribution pipelines $[3,9,26]$, considering all of the frequencies analyses with identical resolutions.

Wavelet transform (WT) is a time-frequency analysis method, which presents excellent time and frequency localization. Since the past two decades, the investigation of the acoustic emission signal with a high capability of wavelet transformation has changed the way of thinking and has brought significant efforts [3,26-31]. A number of studies have demonstrated that the WT can be applied in the analysis of acoustic signals effectively. However, no research has validated an appropriate method for the selection of a suitable mother wavelet despite its importance and effect on their results analysis. Though, from these investigations, it can be concluded that the choice of mother wavelet depends upon the motive of study and nature of features of the signal. Such as Ran Wu [32] has analyzed WT advantages over STFT for the acoustic emission signal detection, Kalogiannak [33] has applied the WT method for the analysis of acoustic emission signals to investigate the defects in composite materials. In this study, due to its simplicity, the Daubechies (db) mother wavelet has been chosen. Lotus [34] has also suggested WT for acoustic signal features identification in composite materials. However, the motivation for the selection of mother wavelet is not manifested. Boczar [35,36] has analyzed the acoustic emissions caused by the electric discharge in air, applying WT without considering the 
significance of choice of the mother wavelet. Ahadi [3] has proposed and emphasized the importance of mother wavelet for leak detection in plastic water pipes. However, the author selected optimal mother wavelet "Gauss27" in the context of shape only; whereas, other correlated mother wavelets are not explored. The features of acoustic leak signals in plastic water pipes are identified by Butterfield [26] by using WT; this study has focused the importance of mother wavelet, though the leakage signatures intervals are not recognized. The aforementioned studies incredibly demonstrate the significance of extracting features of acoustic emission signals that provide valuable information. Besides this, for effective results analysis still, needs a proper guideline to select suitable mother wavelet.

This research aims to purpose a methodology for reliable underwater leak detection in buried long transmission lines. In this study, it is validated that combining the hardware (free-swimming device) and tuned WT (optimal mother wavelet) together can efficiently lead to the detection of hidden leaks and unwanted correlated signals from fickle underwater measured acoustic signals. Moreover, this study confirms that WT tuning can reveal hidden events and also illustrates the significance of choice of mother wavelet for underwater acoustic signals.

The event clustering of underwater measured acoustic signals is performed efficiently by applying the extracted prior knowledge of the signals and the selection of optimal mother wavelet. The proposed methodology comprised of following four main steps.

1. The significance of WT-In this step, it is demonstrated that WT evident to hidden features and the importance of selection of mother wavelet by processing the real-time underwater measured acoustic signal.

2. Acoustic signals signatures classification-Where leakage signatures are extracted under the influence of external and internal pipeline's unwanted environmental signals (instantaneous pipe vibrations, water flow noise, pipe's natural frequencies, and background pump noise) by predefined controlled experiments.

3. Wavelet Tuning-Maximum correlated or optimum mother wavelet is selected for identified leakage signatures.

4. Acoustic signal clustering and detection-In this step, the optimum mother wavelet is applied to detect leakage and related interferences from a cluster of experimentally measured data.

The rest of the paper is organized as follows: Section 2 briefly illustrates the reasons for analysis of underwater acoustic signals in WT. In Section 2.1, a short review of WT and the importance of mother wavelet is described; besides this, from above outlined four main steps, step 1 is validated in Section 2.2. Section 3 demonstrates the general methodology of underwater leakage detection and the selection of the optimum mother wavelet. Section 4 contains the experimental methodology in which test-rig, free-swimming device intelligence, device launching, and controlled experiment conducting method are demonstrated. In Section 5, the rest of the above outlined important steps, step 2 (Section 5.1), step 3 (Section 5.2), and step 4 (Section 5.3) are demonstrated and implemented, respectively. Finally, in Section 6, conclusions and future works are presented.

\section{Underwater Acoustic Signal Processing Methodology in Wavelet Transform}

While inspecting leakage in a plastic water-filled pipe through the free-swimming acoustic device; it has manifested that due to the influence of various factors (water flow, background noise, and pipe's natural frequency) leakage frequency components are completely hidden inside of the underwater acoustic signals. This investigation incredibly demands the extraction or the classification of features of the acoustic signals. Hence, a number of studies have validated that WT produces the excellent time-frequency localization. However, significant issues are leakage evidence and the selection of the best mother wavelet that depends upon both the goal of the study and the features to be analyzed. 


\subsection{Wavelet Transform}

A wavelet is a small oscillating waveform of the limited interval, which is beginning at an amplitude of zero, increasing and then decreasing back to zero value. WT overcomes the time-frequency localization boundaries of Fourier, Shanon, and STFT. One significant point is that the wavelets have the ability to retrieve the information regarding local properties of the signal. A signal with finite energy is signified as mother wavelet, which is expressed as;

$$
\psi_{(a, b)} t=\frac{1}{\sqrt{a}} \psi\left(\frac{t-b}{a}\right), a>0,-\infty<b<\infty
$$

where family $\psi_{(a, b)}$ subsists with all normalized dilations in the time $t$ specified by a scale factor $a>0$ and translations specified by $-\infty<b<\infty$ in time $t$ [37].

Hence, wavelet transform (WT) of specified acoustic signal $f(t)$, is defined regarding cross-correlation with mother wavelet $\psi_{(a, b)}$ as

$$
W T_{f}(a, b) \stackrel{\Delta}{=} \int f(t) \psi_{(a, b)}(t) d t
$$

In case that $f(t)$ is the transient event and composed of elemental waves comparable to $\psi_{(a, b)}$, at times of occurring of elemental waves within the $f(t)$; the wavelet transform $W T_{f}(a, b)$ tends to have significant localized values. Consider $f(t)$ as a desired signal pattern, and identical to one of the family members of mother wavelets for particular values of $a$ and $b$, then the convolution integral uses smaller values, while $a$ and $b$ are varied, as given in Equation (2), this is as the result of the mother wavelets orthogonality property. The particular, $f(t)$ event is also orthogonal to every family member; therefore, corresponding signals can detect the event and localized around the scale factor $a$ and instantaneous time $b$. However, a variety of mother wavelets manifest the events with distinct localization properties and scales. The optimal mother wavelet has an important role in producing the estimable information for the particular study.

\subsection{Significance of Selection of Optimum Mother Wavelet in Underwater Acoustic Signals}

This part of study simply demonstrates the compulsion of WT and the importance of optimum mother wavelet in underwater leakage identification. However, to illustrate the hidden events identification and describing the significance of choice of mother wavelet in non-stationary signals detection, the free-swimming device's real-time measured acoustic signal is investigated.

The experimentally measured acoustic signal $f(t)$, is comprised of various events (instantaneous pipe vibrations, water flow noise, pipe's natural frequencies, and background pump noise) with leak size $6 \mathrm{~mm}$ in the time window $t=0$ to $43 \mathrm{~s}$. Two experiments are presented to validate this study; one with closely related suitable mother wavelet, and the other when mother wavelet is not suitably chosen. The first "db12" mother wavelet is selected as shown in Figure 2a, its shape almost matches with the events inside the real-time acoustic signal. The scalogram of "db12" has detected and revealed the hidden events of $f(t)$, it also demonstrates how signal energy varies over scales regarding the intensity of events, but the time domain signal $f(t)$ cannot manifest these diversifications (see Figure 3a). The WT visual scalogram has illustrated the diversity and the localization of events for the period of $1<t<17 \mathrm{~s}, 18<t<20 \mathrm{~s}$, and $20<t<40 \mathrm{~s}$, respectively. The significant variation in $f(t)$ is ostensibly notable for the interval of $18<t<20 \mathrm{~s}$ and robust frequency components exist at scales $10<a<257$, which perhaps indicate leakage. Besides this, due to the selection of improper mother wavelet as shown in Figure 2b, WT detect little or no similarity to signal events. The visual scalogram of "Haar" mother wavelet [38] could not localize and identify the comprised signal events in the result of the inappropriate choice of the mother wavelet, as shown in Figure $3 \mathrm{~b}$. This analysis confirms that the underwater recorded signal $f(t)$ cannot be evident to the diversification of events inside signal as a result of variations of surrounding parameters. Hence, the free-swimming device and the tuned 
WT along with events localization and detection capability together can lead to attaining reliable and accurate leakage detection in plastic water distribution pipes.

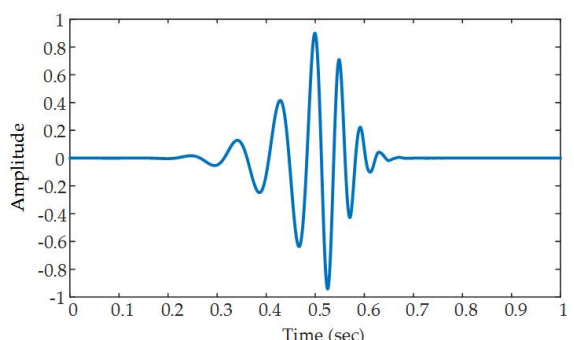

(a)

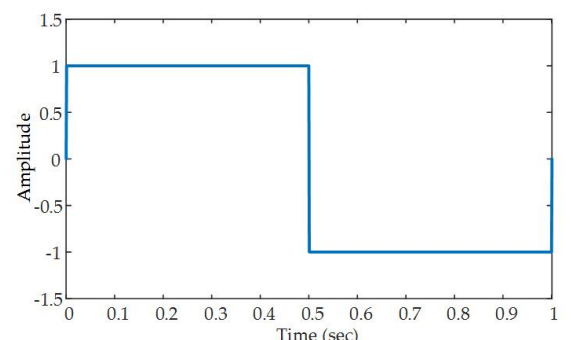

(b)

Figure 2. (a) "db12" mother Wavelet" (b) "Haar" mother wavelet.
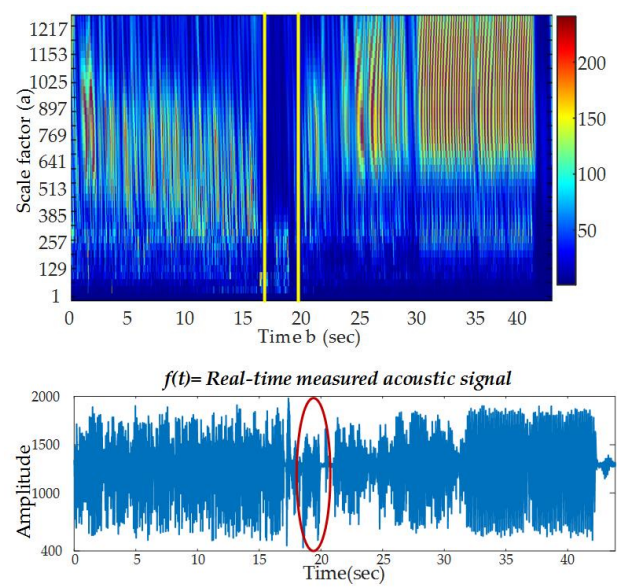

(a)
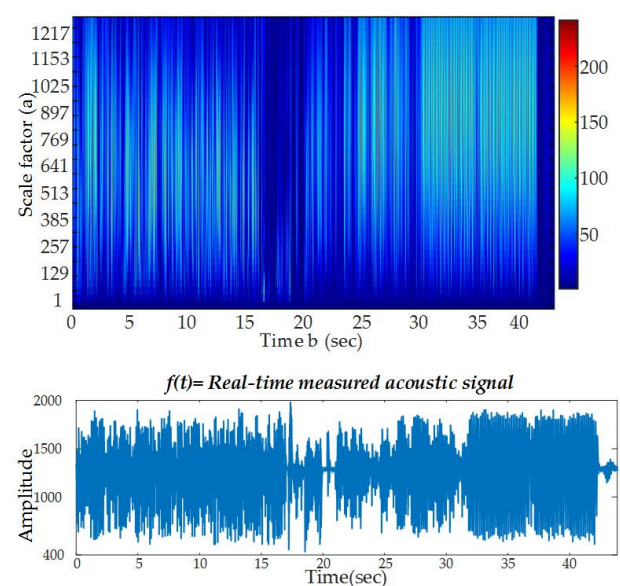

(b)

Figure 3. Localization scalogram of $f(t)$ using (a) db12 "mother Wavelet" (b) "Haar" mother wavelet.

However, due to an enormous number of mother wavelets availability with different localization properties and scales, it provokes to tune the WT to underwater acoustic signals regarding the nature of events. The resemblance of the shape of mother wavelet to the acoustic signal can be the first step in the selection of optimal mother wavelet. Also, it is exhibited that WT tuning can localize and extract the encompassed features of $f(t)$ to generate the estimable information. The aforementioned acoustic signal events (instantaneous pipe vibrations, water flow noise, pipe's natural frequencies, and background pump noise) are investigated in Section 5.

\section{General Method of Underwater Leakage Identification}

The wavelet transform is used to detect the presence of leakage. However, acoustic signals are always embedded in various signals such as; water flow noise, background noise, pipe's natural frequencies, and so forth. Various studies have reported that leaks exist in lower frequency bands of measured acoustic signals in plastic pipes, typically less than $200 \mathrm{~Hz}$ [6,8,39]. For determining the best mother wavelet, some experiments are designed to investigate the characteristics of signals in a standard plastic pipeline and to extract distinction features of leakage acoustic signals, which are referred to as "leakage signatures".

Two necessary steps are taken to identify the leakage signatures. In the first step, it is manifested that the frequency band, wherever the acoustic signal has strong components, correlated to relative interfering comprised noise and signals. Consequently, STFT is tuned (window size) [40-44] to 
non-stationary underwater signals and revealed that the presence of leakage acoustic signal is analogously strong in short-time windows related to other unwanted events (see Figure 4).
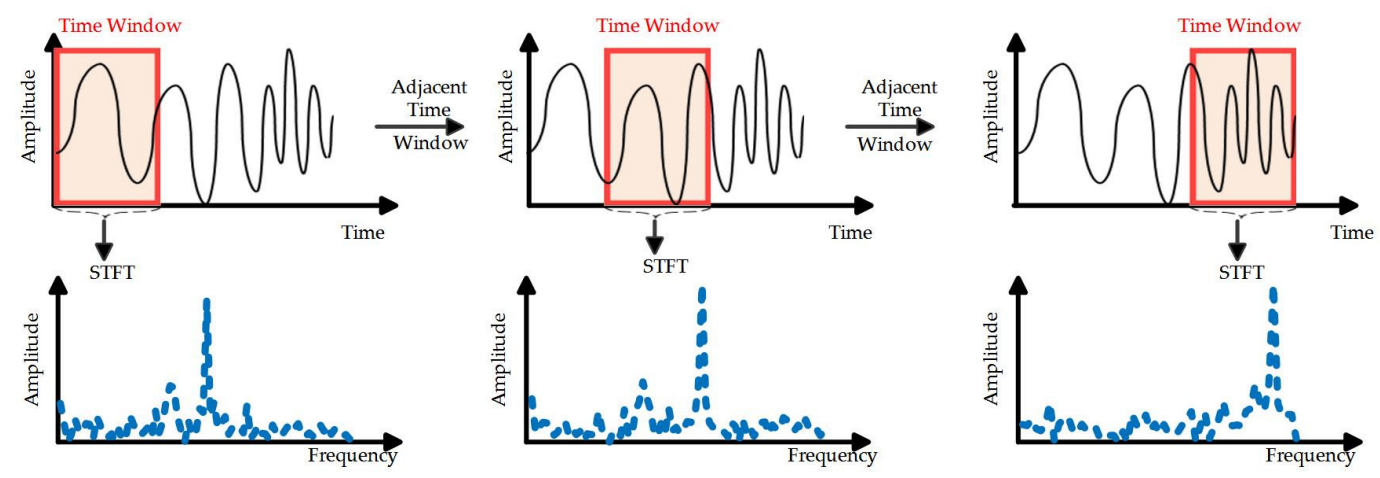

Figure 4. Short-Time Fourier Transform (STFT) sliding time window method.

The underwater acoustic signal is a tangle of multiple events; often it is very challenging to identify the leakage signal. Therefore, to demonstrate leakage signatures, each signal frequency component is realized in the first step. Consequently, STFT's is tuned to exhibit and confine those portions in which leakage components are dominant. It can conclude that the signal in time domain represents the general or concealed shape of underwater acoustic signals. The signal in frequency domain potentially demonstrates the hidden events and correspondingly leads to the selection of appropriate mother wavelet.

\section{Experimental Methodology}

A state of the art laboratory is designed to conduct the underwater experiments by employing a free-swimming acoustic device. The test rig consists of $200 \mathrm{~mm}$ internal diameter, $50 \mathrm{~m}$ long transparent U-PVC material pipe, which comprises of five equally sized $(10 \mathrm{~m})$ segments, installed parallel in a U-shape. The test pipeline can be extended simply by adding more pipe sections of the same or different materials. Launching the device in the line can be difficult, therefore; the unique vertical three-way structure is designed to insert the device correctly into the water pipeline as shown in Figure 5a. The pump is installed on a pipe rig that circulates the water between the water storage tank and pipeline. Initially, two leaks of $20 \mathrm{~mm}$ were created manually at a distance of 10 and $20 \mathrm{~m}$, respectively. To adjust the leak size, the special $4 \times 4$ inch flat metal plates are constructed with different size of holes $(0,4,8,12 \mathrm{~mm}$, etc.), which can be fixed at leak points to regulate the leak size. However, to test the device and simulate leaks, the potentially noisy environment is created for the test rig. The pump is installed very close to the pipeline that generates the constant background noise and vibration in the pipe rig.

After extensive tests of the sensors, the acoustic sensor is chosen based on high underwater sensitivity results. The frequency response range of $20-100 \mathrm{kHz}$ with a sensitivity of $210-220 \mathrm{~dB}$, is capable of detecting the sound of leakage signal. However, to reduce the impact of flow noise and other unwanted signals, preamplifier, and low-pass filter modules are designed to process the sensor signal for enhancing the underwater leakage detection capability of the device. The gain of the preamplifier (AD620, Analog Devices Inc., Norwood, MA, USA) is tuned to 40, and the filtering frequencies are confined by up to $10 \mathrm{kHz}$. A 12-bit Analog to digital converter (ADC) is used to digitize and transmit the recorded acoustic data to the programmed micro-controller (STM32F103), and are finally stored on Kingston SD Card. The high-speed (10 MB/sec) "Class 10" 32 GB SD card is installed to store and access the acoustic data speedily. The Complete circuitry is powered and tested with a rechargeable lithium battery (2000 $\mathrm{mAh}$ ), which can keep the device active for up to $24 \mathrm{~h}$. The free-swimming device circuitry is fully sealed with a spherically shaped aluminum alloy material 
to protect the electronic components from the pipeline environment; its weight is about $0.7 \mathrm{~kg}$. The spherical shape and smaller size $(100 \mathrm{~mm})$ than the pipe bore, support the device to roll smoothly in the complex water line system.

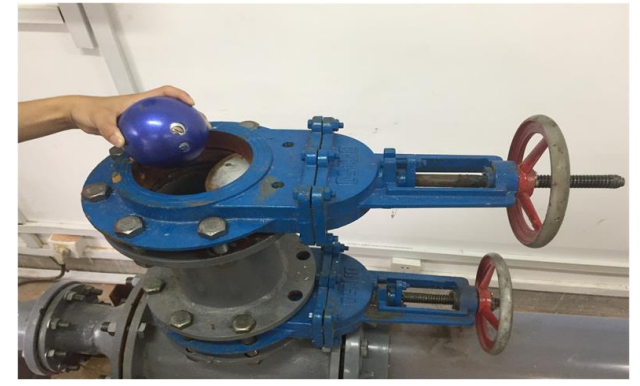

(a)

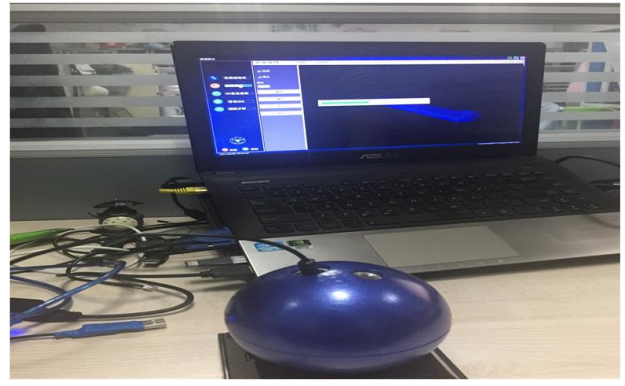

(b)

Figure 5. (a) Free-swimming device launching in three-way structure (b) Acoustic data transferring.

The free-swimming device is launched in a test rig through the appropriate three-way structure to conduct the controlled experiments. A simplified method for acoustic data transfer and charging, which avoids the need of exposing the device circuitry, is shown in Figure 5b. It also has the significance of reducing the risk of water damage to the device's internal components, while the leakage inspection of pipe rig assures that the seals remain unimpaired.

After performing several tests, the minimum flow rate of $0.11 \mathrm{~m}^{3} / \mathrm{s}$ was measured at which the free-swimming device can traverse smoothly in the water pipeline. The device's controlled experiments recorded data is transferred via RS-232 cable to the computer and analyzed using MATLAB (Matrix Laboratory). Underwater testing of the device regarding the memory and battery lastingness has led to the conclusion that the device can analyze approximately $16-18 \mathrm{~km}$ long water transmission lines.

\section{Results and Discussion}

\subsection{Acoustic Signal Signature Identification}

The acoustic signatures identification plays a vital role in the analysis of underwater recorded fickle signals. These signatures become evident to embedded events, reduce the uncertainty regarding false leakage alarms, as well as incredibly lead to the selection of optimum mother wavelet.

Three types of controlled experiments were designed to identify the underwater leakage acoustic signals and unwanted pipeline environmental interfering signals.

Type 1-Background pump noise and pipeline environment: The device is launched to examine the influence of the external and internal pipeline's unwanted environmental signals (instantaneous pipe vibrations, water flow noise, pipe's natural frequencies, and background pump noise) in the absence of leakage signal.

Type 2-Leakage signal: The single small leak size of $6 \mathrm{~mm}$ is regulated by a flat metal plate (discussed in Section 3) at a distance of $10 \mathrm{~m}$ in the presence of aforementioned all-encompassing signals.

Type 3-Leakage size effect: Under the same conditions of the "type 2" experiment, another $10 \mathrm{~mm}$ leak is regulated at a distance of $20 \mathrm{~m}$, respectively, to investigate the influence of leak size, validation of leakage signatures, along with underwater sensitivity, and the inspection capability of the free-swimming device.

The following methodology is applied to predefined experiments measured acoustic data for leakage signature(s) identification and specific time of occurrence, as explained in Section 3.

1. For the analysis of leakage and related interfering noises signatures (frequency components), the Fast Fourier Transform (FFT) is employed first to three types of underwater measured data for 1 $\mathrm{min}$. The analogously long measurement time of $1 \mathrm{~min}$ assures that the all-dominant frequency 
components are in the frequency spectrum. FFT of three types of measured signals is illustrated in Figure 6.

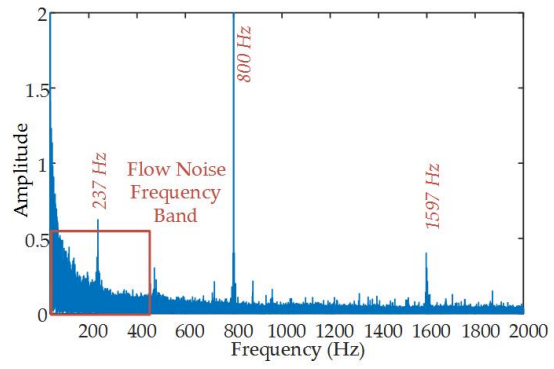

(a)

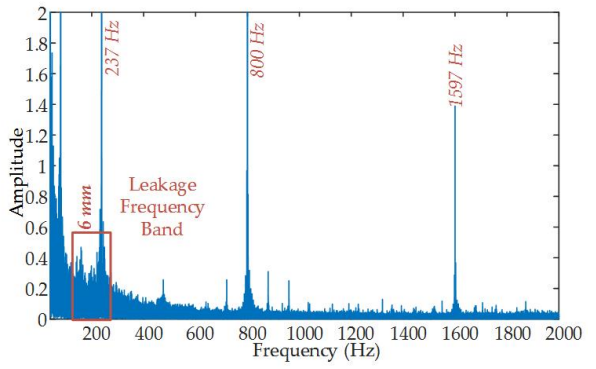

(b)

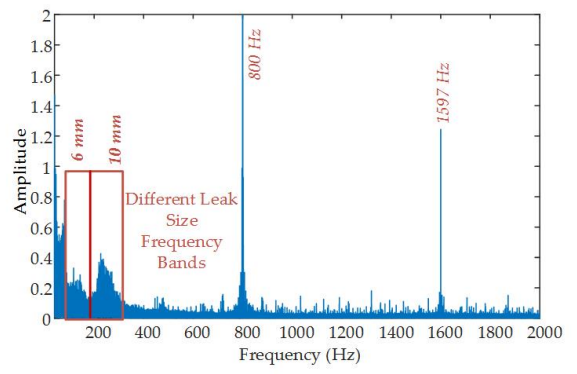

(c)

Figure 6. Fast Fourier Transform (FFT) of measured data comprised of background noise and pipeline environment with (a) no leakage (b) $6 \mathrm{~mm}$ leakage size and (c) $6 \mathrm{~mm}$ and $10 \mathrm{~mm}$ leakage size.

In the absence of leakage (Figure 6a), three prominent spikes (signatures) are realized in the frequency spectrum, 237, 800, and $1597 \mathrm{~Hz}$, respectively. A number of investigations and related studies revealed that these notable signatures occurred as a result of the pump (for example see [45]); which was installed in a test rig near the device launching segment. Therefore, it has generated consistently momentous vibrations. Figure 6 a also incredibly demonstrates that the water flow noise dominating frequency components are between 0 to $400 \mathrm{~Hz}$ approximately, that makes it very challenging to differentiate between the leakage signature and the flow noise in the same frequency band. The impact of the pipe's natural frequencies is not distinctly prominent in the spectrum, possibly due to the prominent flow noise frequency band. The background noise signatures are also not self-evident in the spectrum, which illustrates that external noises cannot be predominant inside the water-filled pipes. Nevertheless, these results validate that the time domain signal is unable to interpret hidden features. The pre-investigation (absence of leakage) of the pipeline environment is the essential fundamental step to inspect the unwanted underwater interferences.

In the presence of small leakages (Figure 6b), as specified in the "type 2" experiment, the noticeable energy change occurs between 100 to $200 \mathrm{~Hz}$ in the lower frequency bands of the spectrum. Since, in the light of various studies and individual experimental investigations, it is observed that usually, the lower frequency bands carry leak signals in plastic pipes. Perhaps this change in energy intimates the leakage. Despite the fact that the "type 3" experiment is designed to authenticate the leakage signatures, leak size impact, and the device's underwater sensitivity capability. The two different sizes of leaks (Figure 6c) has generated the prominent distinct frequency signatures in the range of "100 to 200 " and "210 to $323 \mathrm{~Hz}$ ", respectively in the spectrum. It is also very noteworthy that the frequency band ( $93 \mathrm{~Hz}$ to $200 \mathrm{~Hz}$ ) is similar as the result of "type 2" experiment measured data. The FFT analysis explicitly validated that these significant signatures occurred due to leakage(s) in lower frequency bands. Besides this, the free-swimming acoustic device through the great potential of acoustic sensitivity has incredibly inspected the small underwater leakages in the plastic pipeline. 
However, FFT frequency spectrums of 1 min measured data (Figure 6) have demonstrated the leakage signature(s) and the associated unwanted interferences signatures, still need to confine definite time interval of occurrence that would lead to select the optimal mother wavelet.

2. In this step, "type 1" and "type 2" experimental measured data is chosen to localize the captured acoustic signal signatures, the sliding short-time window is tuned to $25 \mathrm{~ms}$ with a lowest detectable frequency of $40 \mathrm{~Hz}$. The STFT window size is confined to identify the intervals at which leakage signals ( 93 to $323 \mathrm{~Hz}$ ) have robust frequency components occurred regarding the flow noise and other related undesired sources, as shown in Figure 7. In the absence of acoustic leakage signal (Figure 7a), no significant energy change occurred in the spectrogram and flow noise energy was distributed almost homogeneously. However, other related sources high-frequency components are not much apparent as the result of window size limitation to lowest frequencies. Similarly, in the presence of leakage (Figure $7 \mathrm{~b}$ ), notable energy change has appeared in the STFT spectrogram for the period of $18<t<20 \mathrm{~s}$, which certainly intimates the leakage signature. Various studies in most of the cases have set the short time window to $10 \mathrm{~ms}$. Nevertheless, the number of experimental acoustic measured data investigations have revealed that the short time window selection depends upon the features of the signal to be analyzed. It is also noticeable that a $25 \mathrm{~ms}$ time window is determined for STFT that bounds the lowest frequency to $40 \mathrm{~Hz}$, which covers the leakage frequency bands between 100 to $393 \mathrm{~Hz}$. Besides this, it concludes that these signals are the acoustic leakage signatures that will significantly lead to select the optimal mother wavelet in next section.
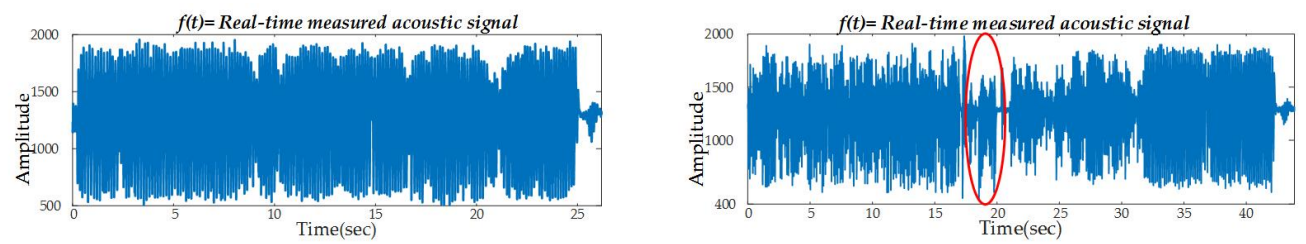

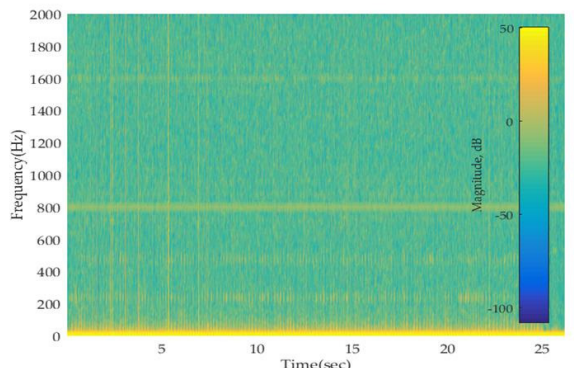

(a)

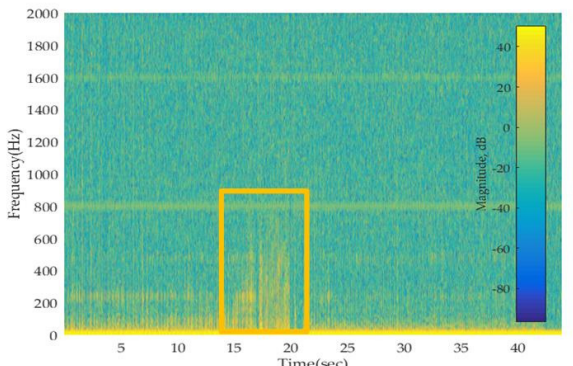

(b)

Figure 7. STFT of measured data comprised of background noise and pipeline environment with (a) no leakage (b) leakage (6 $\mathrm{mm}$ size).

\subsection{Selection of Optimal Mother Wavelet}

Since the three types of experimentally measured data is analyzed, more than one acoustic signal signatures have been revealed. Though these leakage signatures will drive to the selection of best mother wavelet, correspondingly it is also important to figure out which type of the measured data (single or multiple acoustic signatures) should be processed in wavelet transform (WT). However, while examining a large number of experimentally measured data in WT, it has verified that the acoustic signal having more than one dominant acoustic signatures or different events can efficiently lead to the selection of standard mother wavelet. It is remarkable that the acoustic signal having a single acoustic signature or event often misleads the selection of mother wavelet. Therefore aforementioned "type 3" experiment data is analyzed for the choice of the optimal mother wavelet. 
Figure 8 depicts the different visual scalograms of correlated standard mother wavelets of the acoustic signal. While for determining the best mother wavelet, the most consistent wavelets parameters; localization of the signal shape, acoustic leakage signatures, and flow noise higher amplitude coefficients at scale factor "a" are compared and analyzed correspondingly. It is also noteworthy that the color bar represents the intensity of frequency components at scalogram.

The visual inspection shows that "Haar" and "bior3.1" (Figure 8a, b) have not localized the shape and the measured acoustic leakage signatures profoundly in the scalogram. These results incredibly demonstrate the importance of choice of the appropriate mother wavelet. Sequentially, an energy scalogram of "db12", "rbio2.6" and "sym8" (Figure 8c-e) has localized the pre-identified measured leakage signatures between the intervals $16<t<18 \mathrm{~s}$ and $31<t<35 \mathrm{~s}$ at scalogram(s). However, visually the shape does not match the original acoustic signal. Besides this, it has also demonstrated that the scalogram energy distribution or coefficients are not strong among the confined scales of leakage signatures.

Since the "Mexh", "Gaus 8", and "Morlet" (Figure 8f-h) mother wavelets have significantly localized the signal shape and leakage signatures. This information is considerably estimable, though, in the context of choice of the optimum mother wavelet, it is not adequate. Therefore, along with these parameters, intense energy (high-frequency components) in the leakage frequency regions and water flow noise regions are compared regarding higher scale factor " $a$ " to obtain the best mother wavelet (see Table 1).
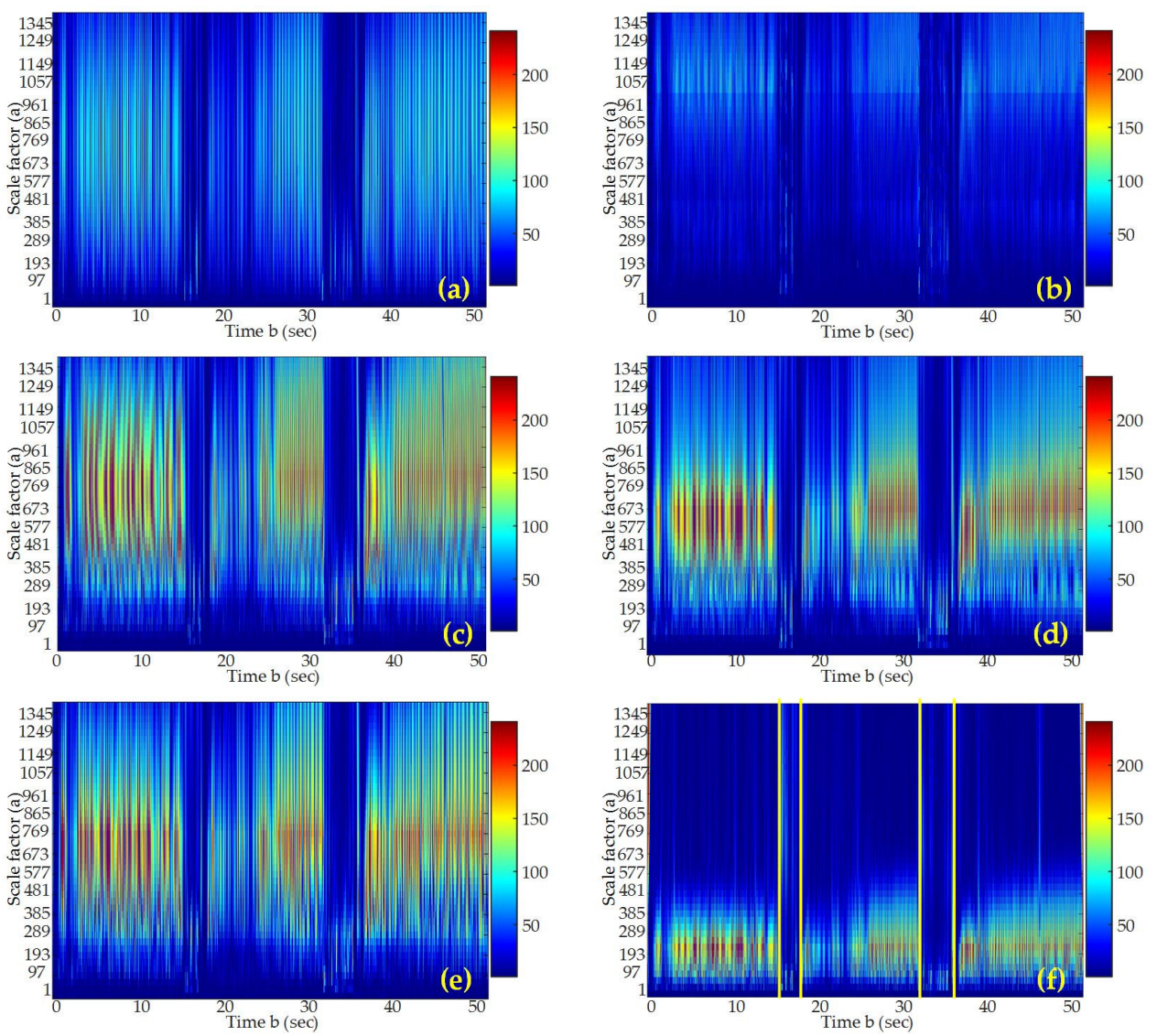

Figure 8. Cont. 

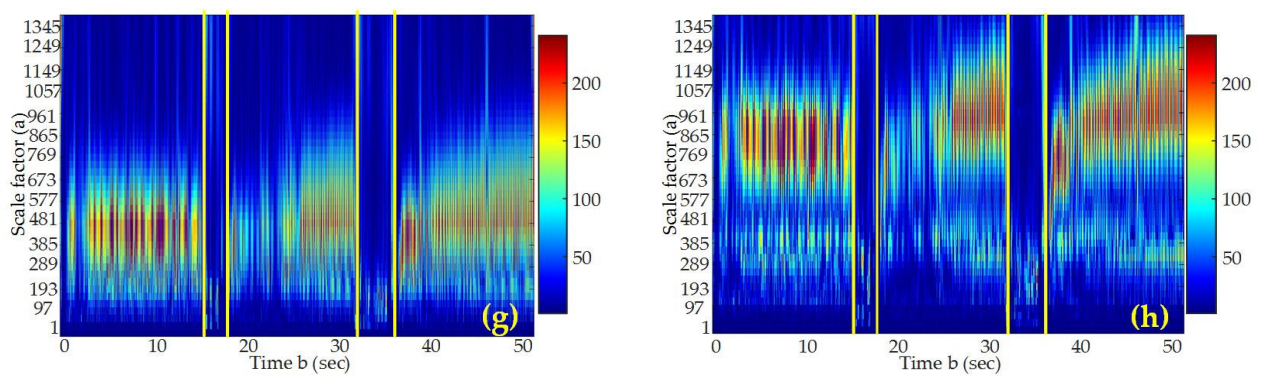

Figure 8. A localization comparison scalogram of the signal shape and leakage signatures using standard mother wavelets, (a) Haar, (b) bior3.1, (c) db12, (d) rbio2.6, (e) sym8, (f) Mexh, (g) Morlet, and (h) Gaus8.

Table 1. The comparison of most correlated mother wavelets parameters regarding the acoustic signal.

\begin{tabular}{cccc}
\hline Mother Wavelets & $\begin{array}{c}\text { Acoustic Signal } \\
\text { Shape Localization }\end{array}$ & $\begin{array}{c}\text { Leakage Frequency Bands } \\
\text { Coefficients Comparison at } \\
\text { Scale Factor }(\boldsymbol{a})\end{array}$ & $\begin{array}{c}\text { Water Flow Noise Regions } \\
\text { Coefficients Comparison at } \\
\text { Scale Factor }(\boldsymbol{a})\end{array}$ \\
\hline Mexh & Average & $20<a<97$ & $97<a<481$ \\
Gauss 8 & Good & $20<a<289$ & $193<a<865$ \\
Morlet & Excellent & $20<a<481$ & $193<a<1345$ \\
\hline
\end{tabular}

In Table 1, the results comparison of the most correlated mother wavelets ("Mexh", "Gaus 8", and "Morlet") parameters, the "Morlet" has demonstrated the leading results. The "Morlet"scalogram (Figure $8 \mathrm{~h}$ ) has presented the excellent localization, leakage frequency bands, and water flow noise energy regions at higher scales $(20<a<481$ and $193<a<1345)$, respectively, in contrast to other associated mother wavelets. Therefore, "Morlet" mother wavelet is chosen an optimal mother wavelet for leakage detection and analysis.

However, instantaneous vibrations traces (Section 5.1) are not prominent at the wavelets scalogram(s) possibly due to the influence of water flow noise or leakage signal. Despite the fact that the presence of these vibration signatures can be examined by analysis of the signal at lower scales in WT or STFT sliding time window, pointing to higher frequencies.

\subsection{Clustering and Acoustic Signal Identification}

The "Morlet" mother wavelet is selected to analyze the cluster of experimentally measured data to detect the leakage, related interferences, and leak size effect. Figure 9 illustrates the scalograms of all three "types" of measured data from the plastic pipeline (see Section 5.1). These measured acoustic data are investigated in preferred appropriate mother wavelet regarding prior knowledge (leakage signatures) of the signals.

The Figure 9a scalogram shows that the pipeline environment, in the absence of leakage signal, has no significant energy change occurred at any instance of time scale. The visual inspection has unveiled that the maximum water flow noise energy is mostly evenly distributed for the parameter values of approximately $577<a<1249$. Besides, it also demonstrates that the instantaneous vibrations without leakage signal for the interval of $1<t<5 \mathrm{~s}$ in lower scales, though aforementioned that these vibration signatures could not be prominent in the presence of the leakage signal(s). These findings validate that the WT localization performance differs event to event for same characteristics of the signal. Therefore, it is noteworthy that the signal should be composed of at least two distinct events that can efficiently lead to optimum mother wavelet. 


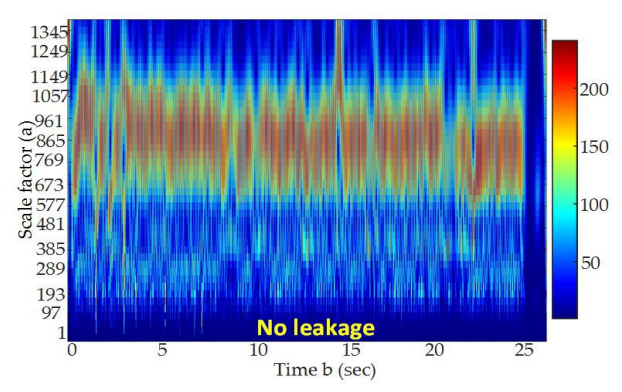

(a)

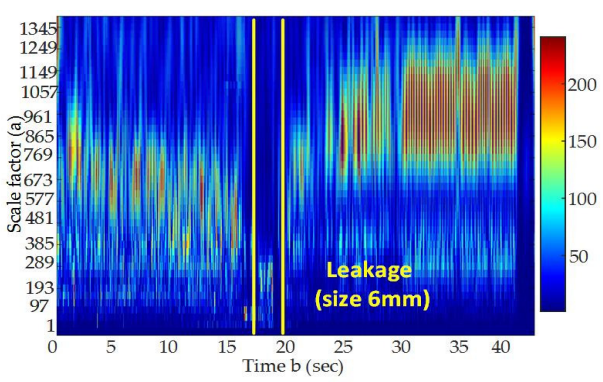

(b)

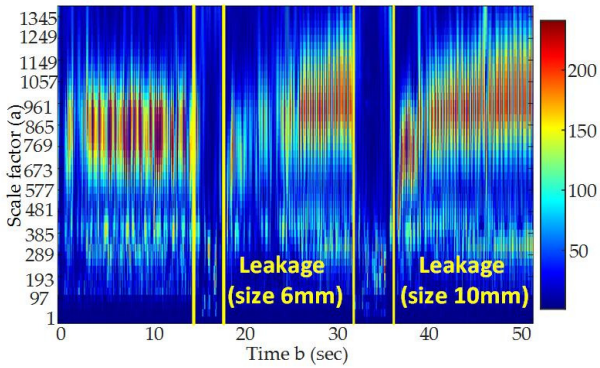

(c)

Figure 9. The scalograms of the measured signals with (a) no leakage, (b) leakage size, $6 \mathrm{~mm}$, and (c) leakage size, $6 \mathrm{~mm}$ and $10 \mathrm{~mm}$.

For investigating the leakage, the small leak size of $6 \mathrm{~mm}$ is regulated at the distance of $10 \mathrm{~m}$ under the influence of the pipeline environment. The three prominent events variation are observed at different time intervals $1<t<17 \mathrm{~s}, 18<t<20 \mathrm{~s}$, and $20<t<40 \mathrm{~s}$, respectively (see Figure $9 \mathrm{~b}$ ). Experimentally, it is realized that these noticeable changes for the interval of $1<t<17 \mathrm{~s}$ and $20<t<40$ have befallen in the resulting instant flow variations. It is noteworthy that the water flow can be unpredictable at any instant in the pipeline; therefore, reliable underwater leakage detection only from measured data is quite challenging. However, regarding prior knowledge, the strong energy region is realized at scales $20<a<481$ for the period of $18<t<20 \mathrm{~s}$, which potentially intimates the leakage.

Subsequently, for the profound investigation and authentication of leakage and the leak size effect, the leak size of $10 \mathrm{~mm}$ is regulated at a distance of $20 \mathrm{~m}$ under the same scenario mentioned above. The Figure 9c scalogram demonstrates the leakages with different leaks size of $6 \mathrm{~mm}$ and $10 \mathrm{~mm}$. Incredibly, the two prominent distinct groups of frequency components regions are realized at scales $20<a<481$ (leakage frequency bands) for the interval of $16<t<18 \mathrm{~s}$ and $31<t<35 \mathrm{~s}$. Both of the strong regions have a similar scales range, despite the fact that the free-swimming device underwater traverses near the leaks locations, therefore, visualizing the time duration (regions width) and calculating leakage frequency bands (see Section 5.1) that can efficiently signify the leak size effect. So, in the light of identified leakage signatures and prior experiment analysis, it can explicitly conclude that the existence of these two strong frequency components regions at scales $20<a<481$ is as a result of leaks.

\section{Conclusions and Future Works}

A free-swimming device with high acoustic sensitivity is capable of detecting the small underwater leakages in the plastic water pipeline. Despite the fact, the experimental analysis of underwater acoustic signals has demonstrated that the leakage frequencies and flow noise frequencies often exist in the same frequency band of the spectrogram. Hence, the classification of the leakage, leak size effect, and the correlated interfering signals from underwater acoustic measured data is considerably difficult. For differentiating the leakage signals, water flow noise, and related unwanted interfering signals, the WT can be tuned to the underwater acoustic signals for localization, leakage detection, and the 
leak size effect. The availability of an enormous number of mother wavelets and their visual results diversity has remarkably demonstrated the importance of the selection of the optimal mother wavelet. The STFT short-time sliding windows are tuned to locate and pinpoint the acoustic leakage signals, which lead to the selection of optimal mother wavelet. Subsequently, the number of acoustic signals investigation have revealed that the wavelets localization results differ event to event for the similar characteristic of the signal. Therefore, the signal, having more than one distinct event is chosen, it also points to the most appropriate mother wavelet. While examining the correlated standard mother wavelet's energy scalograms, the "Morlet" mother wavelet has greatly localized the acoustic signals along with the leakages and the influence of leak size, and their corresponding energy levels. In the selection of optimal mother wavelet, the signal shape localization is the only inadequate information. Therefore, related parameters, flow noise, and leakages of strong frequency components of the most appropriate mother wavelet's scalograms were compared at the scale factor. This study certainly validates the free-swimming device and the tuned WT together can lead to the reliable automatic underwater leakage detection and influence of leakage size in plastic water-filled pipes.

Water pipeline system still demands extremely efficient water management to differentiate and fix the faults and leakages in long buried water transmission lines to protect the environment and bridge the increasing breaches between demand and supply of potable water in urban and rural areas. Hence, the free-swimming device with the strength of tuned WT can be cost effective, reliable, and complement the technology to the existing expertise leak detection systems to reduce these losses. Future work is proposed to investigate the underwater leakages in different types of water pipe materials and the effects of the various type of leaks, such as a joint leaks, etc. Moreover, we will emphasize and improve the location accuracy of the inertial navigation system (INS), which consists of an accelerometer and a gyroscope to recognize the velocity and acceleration, respectively, and the position can be discovered relative to the known starting point.

Acknowledgments: This work was funded by the National Natural Science Foundation of China (No. 61573313) "Online water-quality anomaly detection, classification, and identification based on multi-source information fusion" and (No.U1509208) "Research on big data analysis and cloud service of urban drinking water network safety".

Author Contributions: The designing of the free-swimming acoustic device and underwater leak detection analysis was the complete teamwork project. Dileep Kumar initiated the central idea of the research within the development of his Ph.D. This idea was thoroughly discussed with Dileep's Ph.D. advisors and project team. With the planning, dedicated research, and a collaborative team; Dileep, Dezhan, Naifu, and Dibo Huo designed the free-swimming device, experimental laboratory, and controlled experiments under the supervision of Hongjian Zhang. Dileep was also the in charge of data analyses and this first manuscript draft. Hongjian Zhang and Reehan edited the manuscript and recommended several amendments ultimately leading to the final version.

Conflicts of Interest: The authors declare no conflict of interest.

\section{References}

1. Mekonnen, M.M.; Hoekstra, A.Y. Four billion people facing severe water scarcity. Sci. Adv. 2016, 2, e1500323. [CrossRef] [PubMed]

2. Campbell, E.; Izquierdo, J.; Montalvo, I.; Pérez-García, R. A Novel Water Supply Network Sectorization Methodology Based on a Complete Economic Analysis, Including Uncertainties. Water 2016, 8, 179. [CrossRef]

3. Ahadi, M.; Bakhtiar, M.S. Leak detection in water-filled plastic pipes through the application of tuned wavelet transforms to acoustic emission signals. Appl. Acoust. 2010, 71, 634-639. [CrossRef]

4. Li, R.; Huang, H.; Xin, K.; Tao, T. A review of methods for burst/leakage detection and location in water distribution systems. Water Sci. Technol. 2015, 15, 429-441. [CrossRef]

5. Murvay, P.-S.; Silea, I. A survey on gas leak detection and localization techniques. J. Loss Prev. Process Ind. 2012, 25, 966-973. [CrossRef]

6. Hunaidi, O.; Chu, W.T. Acoustical characteristics of leak signals in plastic water distribution pipes. Appl. Acoust. 1999, 58, 235-254. [CrossRef] 
7. Hunaidi, O.; Wang, A.; Bracken, M.; Gambino, T.; Fricke, C. Acoustic methods for locating leaks in municipal water pipe networks. In Proceedings of the International Conference on Water Demand Management, Dead Sea, Jordan, 30 May 2004; pp. 1-14.

8. Gao, Y.; Brennan, M.J.; Joseph, P.; Muggleton, J.; Hunaidi, O. On the selection of acoustic/vibration sensors for leak detection in plastic water pipes. J. Sound Vib. 2005, 283, 927-941. [CrossRef]

9. Muggleton, J.; Brennan, M. Leak noise propagation and attenuation in submerged plastic water pipes. J. Sound Vib. 2004, 278, 527-537. [CrossRef]

10. Zhang, J. Designing a cost-effective and reliable pipeline leak-detection system. Pipes Pipelines Int. 1997, 42, 20-26.

11. Kurtz, D.W. Developments in a free-swimming acoustic leak detection system for water transmission pipelines. In Proceedings of the Pipeline Division Specialty Conference 2006: Service to the Owner, Chicago, IL, USA, 30 July-2 August 2006.

12. Ariaratnam, S.T.; Chandrasekaran, M. Development of an Innovative Free-Swimming Device for Detection of Leaks in Oil and Gas Pipelines. In Proceedings of the Construction Research Congress 2010: Innovation for Reshaping Construction Practice, Banff, AB, Canada, 8-10 May 2010; pp. 588-596.

13. Chapman, H. Development of a Successful Internal Leak Detection and Pipeline Condition Assessment Technology for Large Diameter Pipes. In Proceedings of the 6th Annual WIOA NSW Water Industry Engineers \& Operators, Tamworth, Australia, 28-29 March 2012.

14. Almazyad, A.S.; Seddiq, Y.M.; Alotaibi, A.M.; Al-Nasheri, A.Y.; BenSaleh, M.S.; Obeid, A.M.; Manzoor Qasim, S. A proposed scalable design and simulation of wireless sensor network-based long-distance water pipeline leakage monitoring system. Sensors 2014, 14, 3557-3577. [CrossRef] [PubMed]

15. Martinsanz, G.P. Sensors for Fluid Leak Detection; Multidisciplinary Digital Publishing Institute: Basel, Switzerland, 2015.

16. Gong, W.; Suresh, M.A.; Smith, L.; Ostfeld, A.; Stoleru, R.; Rasekh, A.; Katherine Banks, M. Mobile sensor networks for optimal leak and backflow detection and localization in municipal water networks. Environ. Model. Softw. 2016, 80, 306-321. [CrossRef]

17. Nasir, A.; Hussain, S.I.; Soong, B.-H.; Qaraqe, K. Energy efficient cooperation in underlay RFID cognitive networks for a water smart home. Sensors 2014, 14, 18353-18369. [CrossRef] [PubMed]

18. Sadeghioon, A.M.; Metje, N.; Chapman, D.N.; Anthony, C.J. SmartPipes: Smart wireless sensor networks for leak detection in water pipelines. J. Sens. Actuator Netw. 2014, 3, 64-78. [CrossRef]

19. Sheltami, T.R.; Bala, A.; Shakshuki, E.M. Wireless sensor networks for leak detection in pipelines: A survey. J. Ambient Intell. Hum. Comput. 2016, 7, 347-356. [CrossRef]

20. Pure Technologies. Smartball for Water and Wastewater Water Mains. Available online: http://www. puretechltd.com/products/smartball/smartball_leak_detection.shtml (accessed on 16 Auggust 2017).

21. Pure Technologies. Sahara Leak \& Gas Pocket Detection. Available online: http:/ /www.puretechltd.com/ products/sahara/sahara_leak_gas_pocket.shtml (accessed on 16 August 2017).

22. Chatzigeorgiou, D.; Youcef-Toumi, K.; Ben-Mansour, R. Design of a novel in-pipe reliable leak detector. IEEE/ASME Trans. Mechatron. 2015, 20, 824-833. [CrossRef]

23. Chatzigeorgiou, D.M. Analysis and Design of an In-Pipe System for Water Leak Detection; Massachusetts Institute of Technology: Cambridge, MA, USA, 2010.

24. Lai, T.T.; Chen, T.; Huang, P.; Chu, H.-H. PipeProbe: A mobile sensor droplet for mapping hidden pipeline. In Proceedings of the 8th ACM Conference on Embedded Networked Sensor Systems, Zürich, Switzerland, 3-5 November 2010; pp. 113-126.

25. Pure Technologies. Pipediver. Available online: http://www.puretechltd.com/products/pipediver/ pipediver-pccp.shtml (accessed on 16 August 2017).

26. Butterfield, J.D.; Collins, R.P.; Beck, S.B. Feature Extraction of Leaks Signals in Plastic Water Distribution Pipes Using the Wavelet Transform. In Proceedings of the ASME 2015 International Mechanical Engineering Congress and Exposition, Houston, TX, USA, 13-19 November 2015. [CrossRef]

27. Suzuki, H.; Kinjo, T.; Hayashi, Y.; Takemoto, M.; Ono, K.; Hayashi, Y. Wavelet transform of acoustic emission signals. J. Acoust. Emiss. 1996, 14, 69-84.

28. Qi, G.; Barhorst, A.; Hashemi, J.; Kamala, G. Discrete wavelet decomposition of acoustic emission signals from carbon-fiber-reinforced composites. Compos. Sci. Technol. 1997, 57, 389-403. [CrossRef]

29. Qi, G. Wavelet-based AE characterization of composite materials. NDT E Int. 2000, 33, 133-144. [CrossRef] 
30. Ni, Q.-Q.; Iwamoto, M. Wavelet transform of acoustic emission signals in failure of model composites. Eng. Fract. Mech. 2002, 69, 717-728. [CrossRef]

31. Chen, C.; Kovacevic, R.; Jandgric, D. Wavelet transform analysis of acoustic emission in monitoring friction stir welding of 6061 aluminum. Int. J. Mach. Tools Manuf. 2003, 43, 1383-1390. [CrossRef]

32. Wu, R.; Liao, Z.; Zhao, L.; Kong, X. Wavelets application on acoustic emission signal detection in pipeline. In Proceedings of the Canadian Conference on the Electrical and Computer Engineering (CCECE 2008), Niagara Falls, ON, Canada, 4-7 May 2008; pp. 001211-001214.

33. Kalogiannakis, G.; van Hemelrijck, D.; Quintelier, J.; de Baets, P.; Degrieck, J. Classification of wavelet decomposed AE signals based on parameter-less self organised mapping. Int. J. Mater. Prod. Technol. 2011, 41, 89-104. [CrossRef]

34. Loutas, T.; Sotiriades, G.; Kostopoulos, V. On the application of wavelet transform of AE signals from composite materials. Proc. Eur. WG AE Berl. 2004, 42, 433-445.

35. Boczar, T.; Zmarzly, D. Application of wavelet analysis to acoustic emission pulses generated by partial discharges. IEEE Trans. Dielectr. Electr. Insul. 2004, 11, 433-449. [CrossRef]

36. Boczar, T. Application of Wavelet Analysis to Acoustic Emission Pulses Generated by Electrical Discharges in Air. In Proceedings of the DGZfP Proceedings EWGAE, Berlin, Germany, 15-17 September 2004.

37. Wornell, G.; Oppenheim, A.V. Signal Processing with Fractals: A Wavelet-Based Approach; Prentice Hall Press: Upper Saddle River, NJ, USA, 1996.

38. Addison, P.S. The Illustrated Wavelet Transform Handbook: Introductory Theory and Applications in Science, Engineering, Medicine and Finance; CRC Press: Boca Raton, FL, USA, 2017.

39. Pal, M.; Dixon, N.; Flint, J. Detecting \& locating leaks in water distribution polyethylene pipes. In Proceedings of the World Congress On Engineering, London, UK, 30 June-2 July 2010.

40. Nisar, S.; Khan, O.U.; Tariq, M. An efficient adaptive window size selection method for improving spectrogram visualization. Comput. Intell. Neurosci. 2016, 2016. [CrossRef] [PubMed]

41. Benesty, J.; Sondhi, M.M.; Huang, Y. Springer Handbook of Speech Processing; Springer Science \& Business Media: Berlin, Germany, 2007.

42. Boashash, B. Introduction to the Concepts of Time-Frequency Signal Analysis and Processing Time-Frequency Signal Analysis and Processing: A Comprehensive Reference Part I; Elsevier: Oxford, UK, 2003.

43. Dutoit, T.; Marques, F. Applied Signal Processing: A MATLABTM-Based Proof of Concept; Springer Science \& Business Media: Berlin, Germany, 2010.

44. Manolakis, D.G.; Ingle, V.K. Applied Digital Signal Processing: Theory and Practice; Cambridge University Press: Cambridge, UK, 2011.

45. Yoon, D.-B.; Park, J.-H.; Shin, S.-H. Improvement of cross-correlation technique for leak detection of a buried pipe in a tonal noisy environment. Nucl. Eng. Technol. 2012, 44, 977-984. [CrossRef] 